\title{
INTENSIDADE DE RALEIO DE FRUTOS EM PESSEGUEIROS 'FLORDAPRINCE' CONDUZIDOS EM POMAR COM ALTA DENSIDADE DE PLANTIO'
}

\author{
JOÃOALEXIO SCARPARE FILHO², KEIGO MINAMI²e RICARDO ALFREDO KLUGE ${ }^{3}$
}

\begin{abstract}
RESUMO - O objetivo deste trabalho foi verificar o comportamento de pessegueiros (Prunus persica (L.) Batsch.) cultivar Flordaprince, conduzidos em pomar com alta densidade (3.333 plantas/ha), submetidos a diferentes intensidades de raleio manual de frutos. Os tratamentos utilizados foram 120, 100 e 80 frutos por planta, e o controle sem raleio (230 frutos por planta). As plantas submetidas ao raleio produziram frutos significativamente maiores e mais pesados do que os do controle. A produção por planta $(\mathrm{kg})$ e a produtividade estimada ( $\mathrm{t} / \mathrm{ha}$ ) foram maiores nas plantas sem raleio, mas a classificação comercial e a receita bruta $(\mathrm{R} \$ / \mathrm{ha})$ desse tratamento foram menores, devido ao menor tamanho e peso dos frutos. Os tratamentos 100 e 80 frutos por planta $(56,52 \%$ e $65,21 \%$ de raleio, respectivamente) apresentaram os melhores resultados.
\end{abstract}

Termos para indexação: raleio manual, desbaste de frutos, produtividade, pomar compacto, Prunus persica.

\author{
INTENSITY OF FRUIT THINNING IN PEACHES 'FLORDAPRINCE' \\ CONDUCTED IN ORCHARD OF HIGH DENSITY
}

\begin{abstract}
The objective of this paper was to test the behavior of peaches (Prunus persica (L.) Batsch.) cultivar Flordaprince carried out in high dense orchard (3,333 plants/ha) submitted to different intensities of hand thinning of fruits. The treatments tested were 120,100 and 80 fruits per plant and without thinning (230 fruits per plant). The thinned plants produced larger and weightier fruits if compared with control plants. The yield per plant $(\mathrm{kg})$ and estimated productivity (ton/ha) were higher in plants not thinned, but marketing classification and gross income were lower in this treatment due to small fruit size and weight. The treatments 100 and 80 fruits per plant $(56.52 \%$ and $65.21 \%$ of thinning, respectively) presented the best results.
\end{abstract}

Index terms: hand thinning, defruiting, productivity, meadow orchard, Prunus persica.

\section{INTRODUÇÃO}

Considerado planta típica de clima temperado, o pessegueiro(Prumus persica (L.) Batsch.) apresenta seu cultivo comercial desde regiões com inverno bastante rigoroso, com 600 a 1.200 horas de frio abaixo de $7,2^{\circ} \mathrm{C}$, até regiões praticamente desprovidas de frio hibernal, com 20 horas de frio abaixo de $7,2^{\circ} \mathrm{C}$ (Barbosa et al., 1990b). O cultivo econômico do

\footnotetext{
${ }^{1}$ Aceito para publicação em 19 de agosto de 1999.

${ }^{2}$ Eng. Agrôn., Dr., Dep. Produção Vegetal, Escola Superior de Agricultura Luiz de Queiroz (ESALQ), Caixa Postal 9, CEP 13418-900 Piracicaba, SP. E-mail: jascarpa@carpa.ciagri.usp.br

${ }^{3}$ Eng. Agrôn., Dr., Dep. Produção Vegetal, ESALQ. E-mail: rakluge@carpa.ciagri.usp.br
}

pessegueiro em algumas regiões paulistas, consideradas não-tradicionais à cultura, deve-se à utilização de materiais melhorados, principalmente pelo Instituto Agronômico de Campinas, no qual, a partir da década de 60, foram desenvolvidas as cultivares aclimatadas às condições de inverno ameno (Barbosa et al., 1990a). Na atualidade, o Estado de São Paulo é o maior produtor de pêssegos de mesa do Brasil.

Na produção de pêssegos de mesa, um dos requisitos para uma boa aceitação pelo mercado consumidor é o tamanho do fruto, que requer dos produtores a utilização de técnicas que aumentem o seu tamanho e possibilitem maiores ganhos financeiros. Nesse aspecto, o raleio é uma prática cultural indispensável e usada em todas as áreas produto- 
ras de pêssegos e outras frutas de caroço, como forma de aumentar a dimensão do fruto, dada a estreita relação existente entre o número de frutos e o seu tamanho (Johnson \& Handley, 1989).

O raleio consiste na retirada do excesso de frutos, em um nível que não prejudique a produtividade, $\mathrm{e}$ apresenta como principais funções aumentar o tamanho do fruto, evitar a quebra de ramos, reduzir os custos da colheita e promover um equilíbrio entre a fase vegetativa e a reprodutiva da planta, evitando assim a alternância de produção (Faust, 1989).

O método de raleio mais comumente utilizado é o manual, uma vez que o raleio químico não apresenta aplicação comercial, dadas as diferentes respostas observadas em distintos climas e cultivares e o número reduzido de produtos químicos eficazes nesta operação (Coutinho, 1994). Para a efetuação do raleio manual, é tomada como base a capacidade produtiva da planta, deixando-se de quatro a cinco frutos por $\mathrm{cm}^{2}$ da área da secção do tronco, ou observando-se a distância de 10 a $15 \mathrm{~cm}$ entre os frutos na planta (Sachs et al., 1984). Este procedimento tem apresentado bons resultados no sistema de condução empregado largamente no pessegueiro, que é o de vaso aberto com três a quatro pernadas principais onde se inserem os ramos produtivos. Agustí et al. (1997) comentam que os melhores efeitos do raleio são alcançados quando $60 \%$ dos frutos são eliminados no desbaste.

A utilização de maiores densidades de plantio do pessegueiro (mais de 1.000 plantas/ha) está sendo implementada em várias regiões do mundo, uma vez que promove maior produção por área e facilita as práticas culturais ao longo do ciclo (Barbosa, 1989). Nesses plantios, há a necessidade de modificação no sistema de condução da planta, visando mantê-la mais compacta e de porte baixo, promovendo, desta maneira, redução no espaçamento entre plantas e linhas (Campo-Dall'Orto et al., 1988). Diversos são os métodos de condução utilizados para o plantio em altas densidades, como: líder central, condução em Y, poda drástica anual e outros (Fallahi, 1992; Walsh, 1992). A escolha do melhor método varia com a cultivar e a região (Barrit, 1995).

$O$ adensamento de plantios de pessegueiro implica alterações no crescimento da planta, e, conseqüentemente, em seu potencial produtivo, uma vez que aumenta a competição interplanta por carboidratos, luz, água e nutrientes. Desta maneira, as práticas culturais comuns no sistema tradicional de condução, principalmente poda e raleio, devem ser adaptadas à nova arquitetura da planta e ao seu ritmo de crescimento (Barrit, 1995).

Uma vez que a adoção da alta densidade de plantio do pessegueiro é relativamente nova, ainda são escassos os trabalhos referentes ao número de frutos que devem ser deixados na planta para que a produção não seja prejudicada e o tamanho do fruto seja satisfatório. Barbosa et al. (1991) observaram, em pomar compacto (4.167 plantas/ha) de pessegueiros 'Tropical' (cultivar bem precoce) e 'Aurora-1' (cultivar precoce), que a manutenção de 30 a 90 frutos por planta incrementou em até $100 \%$ o peso médio dos frutos em relação aos do controle nãoraleado. Concluíram que, para aquela população de pessegueiros, a manutenção de 60 frutos por planta, por ocasião do raleio, promoveu os melhores resultados quantitativos e qualitativos de produção.

Os parâmetros adotados para o desbaste de frutos nos sistemas tradicionais de cultivo não têm sido eficientes nos pomares compactos, e o excessivo número de frutos deixados por planta prejudica drasticamente sua qualidade e seu valor comercial (Barbosa et al., 1990a).

Este trabalho teve como objetivo estudar diferentes intensidades de raleio manual em pessegueiros 'Flordaprince' conduzidos em pomares de alta densidade de plantas, quanto à resposta de tamanho de fruto, produtividade e renda bruta para $o$ produtor.

\section{MATERIAL E MÉTODOS}

O trabalho foi conduzido no pomar experimental do Departamento de Produção Vegetal da ESALQ/USP, em Piracicaba, SP. Foram utilizados pessegueiros 'Flordaprince', com quatro anos de idade, conduzidos em alta densidade e enxertados sobre o pessegueiro 'Okinawa'. O espaçamento utilizado foi $3 \mathrm{~m} \times 1 \mathrm{~m}$, o que corresponde a uma densidade de 3.333 plantas/ha

As plantas foram conduzidas no sistema de líder central, que consistiu na manutenção da produção próxima a um eixo central, sem a formação de pernadas laterais. As plantas foram podadas sistematicamente após as colheitas anteriores, mantendo o eixo principal e encurtando os ramos produtivos para $5-10 \mathrm{~cm}$ de comprimento. 
Os tratamentos consistiram de diferentes intensidades de raleio manual, representados pelo número de frutos deixados por planta, nas seguintes disposições: tratamento 1: sem raleio (controle); tratamento 2: 120 frutos/planta; tratamento 3: 100 frutos/planta; tratamento 4: 80 frutos/planta.

O raleio foi efetuado 35 dias após a floração, antes do endurecimento do endocarpo, variando-se a quantidade de frutos deixados em cada ramo, de dois a quatro frutos/ramo. As plantas que sofreram raleio apresentavam inicialmente um total de 230 frutos/planta, em média, sendo este o número de frutos utilizado no controle (sem raleio).

As variáveis analisadas foram: peso médio do fruto (g); produção média por planta (kg/planta); produtividade estimada (t/ha) e classificação comercial das frutas. Para a classificação comercial das frutas, foi adotado um critério de classificação vigente em setembro de 1997, de acordo com o seu peso: Especial $=$ menos de $35 \mathrm{~g} ; 1 \mathrm{~A}=35$ a $50 \mathrm{~g}$; $2 \mathrm{~A}=50$ a $80 \mathrm{~g} \mathrm{e} 3 \mathrm{~A}=$ mais de $80 \mathrm{~g}$, com os resultados sendo expressos em porcentagem de frutos nas classes. A receita bruta foi calculada a partir dos preços médios pagos aos produtores no mês de setembro de 1997: Especial $=\mathrm{R} \$ 0,28 / \mathrm{kg}$; $1 \mathrm{~A}=\mathrm{R} \$ 0,40 / \mathrm{kg} ; 2 \mathrm{~A}=\mathrm{R} \$ 0,80 / \mathrm{kg}$ e $3 \mathrm{~A}=\mathrm{R} \$ 1,40 / \mathrm{kg}$.

$\mathrm{O}$ delineamento experimental adotado foi o de blocos ao acaso, com quatro tratamentos e cinco repetições, utilizando-se duas plantas por parcela. Os resultados foram submetidos a análise da variância e comparação de médias pelo teste de Tukey a 5\% de probabilidade. Os resultados de porcentagem de frutos nas classes foram transformados em $\sqrt{\mathrm{x}+0,5}$

\section{RESULTADOS E DISCUSSÃO}

Os tratamentos 100 e 80 frutos/planta apresentaram diâmetro e peso médio do fruto significativamente maiores do que os tratamentos 120 frutos/planta e do que o controle (Tabela 1). O peso médio dos frutos das plantas não raleadas foi mais de $30 \%$ inferior ao observado nas plantas com 80 ou 100 frutos, e cerca de $20 \%$ menor do que o verificado nas plantas com 120 frutos. Com relação ao diâmetro médio, os frutos das plantas nãoraleadas foram cerca de $10 \%$ menores do que os frutos de plantas raleadas. Esses resultados comprovam a eficiência do raleio no aumento do tamanho e peso dos frutos, graças à redução da competição entre eles e ao melhor abastecimento de água, nutrientes e fotossintetizados por parte das fontes (Faust, 1989). Resultados semelhantes foram observados por outros pesquisadores, em sistemas de plantio convencionais ou adensado (Salomão et al., 1988; Barbosa et al., 1991; Agustí et al., 1997).

A produção por planta e produtividade estimada foram significativamente maiores nas plantas não-raleadas (Tabela 1), graças ao maior número de frutos presentes (média de 230 frutos/planta). Em comparação com o raleio mais intenso (80 frutos/planta), a produção por planta e a produtividade foram quase duplicadas nas plantas-controle. Entretanto, as plantas sem raleio apresentaram, em geral, classificação comercial inferior à observada nos frutos de plantas com raleio (Tabela 2), e verificou-se maior porcentual de frutos na classe Especial (menos de $35 \mathrm{~g}$ ). Na classe 1A (35 a $50 \mathrm{~g}$ )

TABELA 1. Efeito de diferentes intensidades de raleio de frutos sobre o diâmetro e peso médio dos frutos, produção por planta e produtividade estimada de pessegueiros 'Flordaprince' conduzidos em pomar compacto (3.333 plantas/ha) ${ }^{1}$.

\begin{tabular}{|c|c|c|c|c|}
\hline Tratamento & $\begin{array}{l}\text { Diâmetro médio } \\
\text { do fruto }(\mathrm{cm})\end{array}$ & $\begin{array}{l}\text { Peso médio do } \\
\text { fruto }(\mathrm{g})\end{array}$ & $\begin{array}{l}\text { Produção por } \\
\text { planta }(\mathrm{kg})\end{array}$ & $\begin{array}{l}\text { Produtividade } \\
\text { estimada (t/ha) }\end{array}$ \\
\hline Sem raleio (controle) $)^{2}$ & $4,42 \mathrm{a}$ & $42,08 \mathrm{a}$ & $9,66 \mathrm{a}$ & $32,20 \mathrm{a}$ \\
\hline 120 frutos/planta & $4,72 b$ & $52,07 \mathrm{~b}$ & $6,25 b$ & $20,83 b$ \\
\hline 100 frutos/planta & $4,98 \mathrm{c}$ & $60,84 \mathrm{c}$ & $6,08 b c$ & $20,26 b c$ \\
\hline 80 frutos/planta & $5,12 \mathrm{c}$ & $66,33 \mathrm{c}$ & $5,30 \mathrm{c}$ & $17,68 \mathrm{c}$ \\
\hline C.V. $(\%)$ & 1,60 & 4,68 & 5,36 & 5,36 \\
\hline
\end{tabular}

${ }^{1}$ Médias seguidas de mesma letra, na coluna, não diferem entre si pelo teste de Tukey, a $5 \%$ de probabilidade

2 Nas plantas sem raleio foram colhidos $230 \pm 5$ frutos. 
não houve diferença entre plantas não-raleadas e aquelas que permaneceram com 120 frutos, em decorrência da proximidade dos pesos médios dos frutos desses dois tratamentos. Na classe $2 \mathrm{~A}$ (50 a $80 \mathrm{~g}$ ), onde se concentraram os maiores porcentuais referentes às plantas raleadas, houve diferença significativa entre plantas raleadas ou não.

As classes $2 \mathrm{~A}$ e $3 \mathrm{~A}$ são as que apresentam a maior demanda de consumo no mercado. Verificouse que as plantas que permaneceram com 100 ou 80 frutos produziram mais de $80 \%$ dos frutos nas classes $2 \mathrm{~A}$ e $3 \mathrm{~A}$ somadas. Em contrapartida, as plantas que permaneceram com 120 frutos e as plantas-controle (sem raleio) apresentaram $50,45 \%$ e $19,84 \%$, respectivamente, na soma das duas classes.

O pessegueiro não apresenta alta alternância de safra, e o raleio é necessário apenas para aumentar o tamanho dos frutos; porém, o raleio invariavelmente reduz o rendimento da colheita em número de frutos por planta, e o incentivo econômico para adotá-lo pode não ser aceito pelo produtor (Faust, 1989). No entanto, ao calcular-se a receita bruta com base na classificação dos frutos (Tabela 3), observa-se que a maior produção e produtividade das plantas sem raleio não resultaram em maiores ganhos financeiros que os tratamentos 100 e 80 frutos/planta, em virtude do tamanho reduzido dos frutos cuja classificação reduz o valor de comercialização. Além disso, os pêssegos das classes 1A e Especial, bastante freqüentes no tratamento sem raleio, normalmente apresentam instabilidade no mercado, no que se refere ao preço e à demanda. A exigência do consumidor é cada vez maior quanto às classes $2 \mathrm{~A}$ e $3 \mathrm{~A}$, e quando ocorre aumento na oferta desses pêssegos, é comum verificar-se queda nos preços das categorias inferiores Especial e 1A, dificultando suas vendas.

Considerando que o custo do raleio em sistema de condução e densidade convencional é de $\mathrm{R} \$ 300,00 /$ ha (Agrianual 99, 1999), conjectura-se que o custo da operação de raleio em pomar com 3.333 plantas/ha, conduzido em sistema de líder central, seja de $\mathrm{R} \$ 500,00 / \mathrm{ha}$. As diferenças em receita bruta, entre as plantas não raleadas e as que permaneceram com 80 ou 100 frutos/planta, são de

TABELA 2. Efeito de diferentes intensidades de raleio de frutos sobre a classificação comercial de pêssegos 'Flordaprince' conduzidos em pomar compacto (3.333 plantas/ha) ${ }^{1}$.

\begin{tabular}{|c|c|c|c|c|}
\hline \multirow[t]{2}{*}{ Tratamento } & \multicolumn{4}{|c|}{ Classes } \\
\hline & Especial (< $35 \mathrm{~g})$ & $1 \mathrm{~A}(35-50 \mathrm{~g})$ & $2 \mathrm{~A}(50-80 \mathrm{~g})$ & $3 \mathrm{~A}(>80 \mathrm{~g})$ \\
\hline & 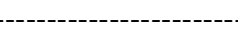 & $---(\%$ de frutc & es) --------- & --------------- \\
\hline Sem raleio (controle) $)^{2}$ & $26,08 \mathrm{a}$ & $51,08 \mathrm{a}$ & $18,81 \mathrm{a}$ & $1,03 \mathrm{a}$ \\
\hline 120 frutos/planta & $3,13 b$ & $46,43 a$ & $48,01 b$ & $2,44 \mathrm{a}$ \\
\hline 100 frutos/planta & $0,65 b$ & $16,13 b$ & $80,11 \mathrm{c}$ & $3,11 \mathrm{a}$ \\
\hline 80 frutos/planta & $0,00 \mathrm{~b}$ & $14,06 \mathrm{~b}$ & $77,32 \mathrm{c}$ & $11,70 \mathrm{~b}$ \\
\hline C.V. (\%) & 33,42 & 16,67 & 6,09 & 32,57 \\
\hline
\end{tabular}

${ }^{1}$ Médias seguidas de mesma letra, na coluna, não diferem entre si pelo teste de Tukey, a $5 \%$ de probabilidade

2 Nas plantas sem raleio foram colhidos $230 \pm 5$ frutos

TABELA 3. Efeito de diferentes intensidades de raleio de frutos sobre a receita bruta/ha, de acordo com a classificação comercial, em pessegueiros 'Flordaprince' conduzidos em pomar compacto (3.333 plantas/ha).

\begin{tabular}{llcccc}
\hline Tratamento & \multicolumn{5}{c}{${\text { Receita bruta }(\mathrm{R} \$ 1.000 / \mathrm{ha})^{1}}^{1}$} \\
\cline { 2 - 5 } & $\begin{array}{c}\text { Especial } \\
(<35 \mathrm{~g})\end{array}$ & $\begin{array}{c}1 \mathrm{~A} \\
(35-50 \mathrm{~g})\end{array}$ & $\begin{array}{c}2 \mathrm{~A} \\
(50-80 \mathrm{~g})\end{array}$ & $\begin{array}{c}3 \mathrm{~A} \\
(>80 \mathrm{~g})\end{array}$ & Total \\
\hline Sem raleio (controle) & 2,357 & 6,586 & 4,847 & 0,162 & 13,952 \\
120 frutos/planta & 0,198 & 3,856 & 8,124 & 0,690 & 12,868 \\
100 frutos/planta & 0,036 & 1,332 & 13,176 & 0,890 & 15,434 \\
80 frutos/planta & 0,000 & 1,042 & 11,465 & 3,025 & 15,532 \\
\hline
\end{tabular}

${ }_{1}$ Preço médio pago no mês de setembro de $1997(\mathrm{R} \$ / \mathrm{kg})$ : Especial $=0,28 ; 1 \mathrm{~A}=0,40 ; 2 \mathrm{~A}=0,80 ; 3 \mathrm{~A}=1,40$. 
$\mathrm{R} \$ 1.580,00$ e $\mathrm{R} \$ 1.482,00$, respectivamente. Tais diferenças são suficientes para cobrir os gastos com o raleio e promover o aumento na receita líquida do produtor.

Os tratamentos 100 e 80 frutos/planta corresponderam ao raleio de $56,52 \%$ e $65,21 \%$ dos frutos, respectivamente, o que concorda com os estudos realizados por Agustí et al. (1997), os quais verificaram que a retirada de $60 \%$ dos frutos resultou na obtenção de frutos maiores em pessegueiros conduzidos em densidades convencionais. Isto permite inferir que, quanto à intensidade de raleio, o pessegueiro cultivado em alta densidade apresenta comportamento semelhante ao verificado em densidade convencional.

\section{CONCLUSÕES}

1. A ausência de raleio em pessegueiro 'Flordaprince' conduzido em pomar com alta densidade promove maior produtividade, porém os frutos produzidos são pequenos e de baixa aceitação no mercado.

2. A técnica de raleio deixando-se 80 ou 100 frutos por planta promove a obtenção de frutos com maiores tamanhos, resultando em maior receita bruta para os produtores.

\section{REFERÊNCIAS}

AGRIANUAL 99: anuário da agricultura brasileira. São Paulo : FNP Consultoria \& Comércio, 1999. 521p.

AGUSTÍ, M.; JUAN, M.; ALMEDA, V.; ANDREU, I.; SPERONI, C. Estímulo del desarrollo de los frutos de hueso. Valência : Generalidad Valenciana/ Consellería de Agricultura, Pesca y Alimentación, 1997. $78 \mathrm{p}$

BARBOSA, W. Desenvolvimento vegetativo e reprodutivo do pessegueiro em pomar compacto sob poda drástica anual. Piracicaba : ESALQ, 1989. 154p. Dissertação de Mestrado.

BARBOSA, W.; CAMPO-DALL'ORTO, F.A.; OJIMA, M.; MARTINS, F.P.; IGUE, T. O pessegueiro no sistema de pomar compacto. IV. intensidade e época de raleio dos frutos dos cultivares Tropical e Aurora-1. Bragantia, Campinas, v.50, n.1, p.93-102, 1991
BARBOSA, W.; CAMPO-DALL'ORTO, F.A.; OJIMA, $\mathrm{M}$. O pessegueiro no sistema de pomar compacto. V. Pesquisas do Instituto Agronômico da década de 80. O Agronômico, Campinas, v.42, n.1, p.35-44, 1990a.

BARBOSA, W.; CAMPO-DALL'ORTO, F.A.; OJIMA, M.; SAMPAIO, V.R.; BANDEL, G. Ecofisiologia do desenvolvimento vegetativo e reprodutivo do pessegueiro em região subtropical. Campinas : Instituto Agronômico, 1990b. 37p. (IAC. Documentos, 17).

BARRIT, B.H. Intensive orchard plantations systems for temperate fruit: consideration and restraints. Revista Brasileira de Fruticultura, Cruz das Almas, v.17, p.1-13, 1995. Número extra.

CAMPO-DALL'ORTO, F.A.; OJIMA, M.; BARBOSA, W.; MARTINS, F.P.; RIGITANO, O. Clones de ameixeira como porta-enxerto ananicante para pessegueiro. Campinas : Instituto Agronômico, 1988. 19p. (IAC. Boletim Técnico, 122).

COUTINHO, E.F. Efeito da cianamida hidrogenada no raleio químico de gemas florais de pessegueiro (Prunus persica (L.) Batsch.) cv. Diamante. Pelotas : UFPEL-Faculdade de Agronomia Eliseu Maciel, 1994. 51p. Dissertação de Mestrado.

FALLAHI, E. Influence of various training systems on yield and fruit quality of low-chill peaches. Acta Horticulturae, Leuven, n.322, p.283-290, 1992.

FAUST, M. Physiology of temperate zone fruit trees New York: J. Wiley, 1989. 338p.

JOHNSON, R.S.; HANDLEY, D.F. Thinning response of early and late-season peaches. American Society for Horticultural Science Journal, Alexandria, v.114, p.852-855, 1989

SACHS, S.; HERTER, F.G.; NAKASU, B.H.; RASEIRA, M.C.B.; FELICIANO, A.G; CAMELLATO, D.; MEDEIROS, A.R.M.; RASEIRA, A.; FONSECA, V.O; PEREIRA, J.F.M.; FINARDI, N.L.; MAGNANI, M.; FEHN, L.M.; SALLES, L.A.B.; FELICIANO, A.; CANTILLANO, R.F.F.; SPERRY, S. A cultura do pessegueiro. Pelotas : EmbrapaCNPFT, 1984. 156p. (Embrapa-CNPFT. Circular Técnica, 10)

SALOMÃO, L.C.; PINHEIRO, R.V.; CONDÉ,A.R.; SOUZA, A.C.G. Efeitos do desbaste manual de frutos na produtividade e na qualidade dos frutos de pessegueiros (Prunus persica (L.) Batsch.), cultivar Talismã Revista Ceres, Viçosa, v.35, n.202, p.596-608, 1988

WALSH, C.S. An overview of peach training systems and the application of pruning techniques. Acta Horticulturae, Leuven, n.322, p.93-98, 1992. 